\title{
Del trabajo cooperativo al trabajo colaborativo: el rol del líder
}

\author{
María Luisa Carrió Pastor $^{\mathrm{a}}$, Hanna Skorczynska ${ }^{\mathrm{b}}$ y Carmen Soler Monreal ${ }^{\mathrm{c}}$ \\ a, b y c Grupo GECOLER. Departamento de Lingüística Aplicada. Universitat Politècnica de València.
}

\begin{abstract}
The main objective of the activity presented in this paper is to practise the competence called effective communication. The learning results were analysed considering written and oral skills. This experimental approach made us consider the way in which students collaborate in teamworks and this is why in this paper we focus on the competences of teamwork and leadership. The activity that we describe has been carried out during the academic year 2015-16 in two faculties at Universitat Politècnica de València: Escuela Técnica Superior de Ingeniería Informática and Facultad de Administración y Dirección de Empresas, with the subjects: "Inglés intermedio-alto para la informática", an optional subject that lasts 90 hours and is scheduled in the fourth year of the degree, and "Inglés para finanzas", taught at Máster en Dirección Financiera y Fiscal. The activity presented has focused on preparing an oral presentation in English about a specific topic using the tools PoliformaT and Google Docs.
\end{abstract}

Keywords: collaborative learning, English for specific purposes, cooperation, leadership.

\begin{abstract}
Resumen
El objetivo principal con el que esta actividad fue diseñada fue trabajar la competencia transversal que se denomina comunicación efectiva. Los resultados de aprendizaje se analizaron tanto a nivel escrito como oral. Esta experiencia nos ha llevado a reflexionar sobre la manera en que los alumnos trabajan en equipo, por lo que en este artículo nos centraremos en las competencias de trabajo en equipo y liderazgo. La actividad que presentamos se ha desarrollado durante el curso 2015-16 en dos centros de la Universitat Politècnica de València: la Escuela Técnica Superior de Ingeniería Informática y la Facultad de Administración y Dirección de Empresas, en las asignaturas "Inglés intermedio-alto para la informática", una asignatura optativa de grado con una duración de 90 horas para alumnos de $4^{\circ}$ curso, e "Inglés para finanzas", impartida en el Máster en Dirección Financiera y Fiscal. La actividad ha consistido en preparar una presentación/exposición formal en inglés sobre un tema de especialidad utilizando Tareas y Chat de PoliformaT y Google Docs.
\end{abstract}

Palabras clave: aprendizaje colaborativo, inglés específico, cooperación, liderazgo. 


\title{
1. Introducción
}

Este artículo aborda varios aspectos que se han tenido en cuenta para el correcto diseño y planificación de las actividades que forman parte del experimento. Por un lado, abordamos las diferencias entre el aprendizaje cooperativo (McCaffert, Jacobs y DaSilva Iddings 2006) y el aprendizaje colaborativo (Nunan 1992, Burns 1999). Así mismo, tenemos en cuenta los conceptos de aprendizaje en equipo y de liderazgo, estudiando los roles de cada uno de estos conceptos en el aprendizaje cooperativo y colaborativo (Carrió Pastor 2006). El aprendizaje colaborativo persigue que los alumnos alcancen madurez, experiencia y que sobre todo se comuniquen con sus compañeros (Storch 2008; Liaw, Chen y Huang 2008; Kessler, Bikowski y Boggs 2012). Este último aspecto es de vital importancia para la enseñanza de lenguas extranjeras. Los alumnos tienen la posibilidad de desarrollar sus propias estrategias de aprendizaje y selccionar sus objetivos de forma colectiva, así como elaborar su propio material (Carrió Pastor 2008, 2009). La interdependencia y respeto mutuo de los componentes del grupo es esencial para obtener una propuesta coherente y avanzar en la adquisición de conocimiento. Los alumnos y los profesores forman un grupo que colabora y negocia en una clase dinámica con un fin último: mejorar el conocimiento.

Nunan (1992: 1) considera que existen las siguientes razones para colaborar:

\begin{abstract}
In language education, teachers, learners, researchers and curriculum specialists can collaborate for a number of reasons. They may wish to experiment with alternative ways of organizing teaching and learning; they may be concerned with promoting a philosophy of cooperation rather than competition; they may wish to create an environment in which learners, teachers and researchers are teaching and learning from each other in an equitable way [...]; or they may wish to experiment with ways of incorporating principles of learner-centredness into their programs.
\end{abstract}

En definitiva, entendemos, como Roschelle \& Teasley (1995: 70) que la colaboración en el aula ha de ser entendida como "[...] the mutual engagement of participants in a coordinated effort to solve the problem together". De esta forma, el aprendizaje colaborativo ha de estar bien planificado y contar con la guía del profesor, ya que la colaboración ha de ser una forma de aprendizaje, no solamente de comunicación entre pares.

El método colaborativo se ha entendido tradicionalmente de dos formas: por una parte, cuando profesores de distintas áreas colaboran para elaborar material o enseñar a alumnos una materia que incluye distintos tipos de conocimiento $\mathrm{y}$, por otra, cuando los alumnos trabajan entre pares los ejercicios que se proponen en clase. En la primera modalidad, el alumno tiene un rol pasivo, siendo el profesor el que propone las actividades, mientras que en la segunda, el alumno es activo y trabaja con otros compañeros para mejorar sus conocimientos, en tanto que el profesor se mantiene al margen de este proceso para procurar que los alumnos sean autónomos y aprendan de forma colaborativa. Como indican Carrió Pastor y Perry (2010) "Collaboration implies interaction among the different members of the group and the different proposals should act as webs of knowledge that combine to offer unique results (Strijbos, Martens and Jochems, 2004: 403)". Es decir, la

(cc)) EY-NC-ND 2016, Universitat Politècnica de València 
comunicación adquiere un papel principal mientras que el liderazgo se queda relegado para provocar que los alumnos interactúen entre sí (Carrió Pastor y Skorczynska 2015).

Por otro lado, el aprendizaje cooperativo se basa en la la interdependencia positiva y la capacidad individual de aprendizaje de sus miembros (McCaffert, Jacobs y DaSilva Iddings 2006), aunque algunos autores lo equiparan al aprendizaje colaborativo (Chung 1991, Romney 1997). Se distinguen en que parece que el término más utilizado para la enseñanza primaria y secundaria entre pares es el aprendizaje cooperativo, mientras que el aprendizaje colaborativo se utiliza más en practicas con alumnos universitarios. En este trabajo, entendemos que los alumnos universitarios que son foco de este estudio inician su aprendizaje entre pares desde una perspectiva cooperativa pero rápidamente se adaptan al aprendizaje colaborativo, ya que les parece más cómodo y las herramientas de las que disponen en sus clases les permiten interactuar de forma colaborativa. Existen estudios que indican que la enseñanza cooperativa respeta en mayor medida el papel de un líder para planificar las actividades, mientras que la enseñanza colaborativa trata a todos los integrantes del grupo como iguales (McCaffert, Jacobs y DaSilva Iddings 2006: 24), pero en nuestro análisis partimos del concepto de colaboración en cuanto todos los miembros forman parte del grupo de forma equitativa pero entendemos que siempre existe un alumno que tiende a planificar y coordinar el grupo y éste será nuestro foco de atención.

\section{Objetivos}

El objetivo principal con el que esta actividad fue diseñada iba dirigido a trabajar la competencia transversal CT-8, la denominada comunicación efectiva. Los resultados de aprendizaje se evaluaron tanto a nivel escrito como oral. Sin embargo, la experiencia también nos ha llevado a reflexionar sobre la manera en que los alumnos trabajan en equipo, por lo que en esta presentación nos centraremos en la competencia CT-6 que es la que versa sobre trabajo en equipo y liderazgo. Por ello, nos planteamos las siguientes preguntas en este estudio: ¿De qué manera se podría reforzar el trabajo colaborativo en un grupo cuando sus miembros realizan tareas individuales? ¿Qué papel puede desempeñar el líder del grupo para reforzar el trabajo colaborativo? ¿Cómo crear una visión positiva del liderazgo por parte de los alumnos?

\section{Desarrollo de la innovación}

La actividad que presentamos se ha desarrollado durante el curso 2015-16 en dos centros: la Escuela Técnica Superior de Ingeniería Informática (ETSIInf) y la Facultad de Administración y Dirección de Empresas (FADE), para las asignaturas "Inglés intermedio alto para la informática", una asignatura optativa de grado de 90 horas para alumnos de $4^{\circ}$ curso de ETSIInf, e "Inglés para Finanzas", impartida en el Máster en Dirección Financiera y Fiscal, impartida en FADE. En el primer caso, se ha trabajado con 21 alumnos organizados en siete grupos de tres. En el segundo caso, se ha trabajado con 22 alumnos en seis grupos de tres y un grupo de cuatro alumnos. La actividad ha consistido en preparar

2016, Universitat Politècnica de València

Congreso In-Red (2016) 
una presentación/exposición formal en inglés sobre un tema de especialidad utilizando las secciones "Tareas" y "Chat" de PoliformaT y Google Docs. La actividad se desarrolla en el aula de informática (en tres sesiones de 1 hora y 30 minutos a lo largo de tres semanas y en dos sesiones de dos horas presenciales cada una durante dos semanas seguidas) y se organiza en tres etapas.

En la primera, el profesor presenta la tarea y se trabaja con materiales que ayuden a preparar una buena presentación en Power Point y su exposición oral. Los alumnos son organizados en grupos de tres. No hay inconveniente en que ellos mismos se agrupen con los compañeros de su elección. Tienen que estar alejados físicamente en el aula para poder reproducir una comunicación remota en situaciones de trabajo colaborativo en empresa. A continuación, el profesor da instrucciones sobre la utilización del chat de PoliformaT para la comunicación entre los miembros de cada grupo/sala y sobre la elaboración del Power Point conjunto utilizando Google Docs. Se presentan varias líneas temáticas relacionadas con informática/finanzas de entre las que los diferentes grupos deberán concretar para su presentación. Utilizando el inglés como única lengua de comunicación permitida durante la actividad, los miembros de cada grupo definen el tema, lo estructuran, planifican la presentación formal y distribuyen tareas a través del chat de PoliformaT. La elección del líder se realiza una vez presentadas las instrucciones para realizar las tareas y después del primer contacto entre todos los miembros del grupo en el chat.

En la segunda etapa, cada grupo hacen una búsqueda de información en internet y elabora una presentación en Power Point en inglés utilizando Google Docs. El líder del grupo envía el documento (Power Point con notas) al profesor como tarea de PoliformaT para su corrección. El profesor devuelve la tarea con un código de corrección y el grupo debe revisar su trabajo y reenviarlo al profesor.

En la tercera etapa, cada grupo de la ETSIInf expone el trabajo corregido al conjunto de la clase. En el caso del Máster en Dirección Financiera y Fiscal, los alumnos graban su presentación en vídeo.

Dentro de la actividad se evalúan tres elementos:

- Participación activa en el chat.

- Documento en Power Point y el texto de la presentación oral. El trabajo es revisado y evaluado dos veces por el profesor como una actividad de producción escrita que supone un $25 \%$ de toda la nota de la producción escrita. La puntuación es común a todos los miembros del grupo.

- Presentación oral/en vídeo. El profesor evalúa la expresión oral de cada alumno, que supone el $20 \%$ de toda la nota de la expresión oral. Los alumnos evalúan aspectos relacionados con la exposición.

Durante el proceso (seguimiento) el profesor observa el funcionamiento de los equipos e interviene para proporcionar ayuda relacionada con las dudas sobre la organización de los contenidos o guiando para el eficaz funcionamiento del equipo registrando estas observaciones en algún instrumento. Al final del proceso de trabajo el profesor evalúa la

(cc)) EY-NC-ND 2016, Universitat Politècnica de València 
adquisición de CT8 (trabajo escrito y oral: claridad, corrección, fluidez, actitud) por parte de los alumnos. Los alumnos evalúan aspectos formales de la presentación de cada grupo (atractiva, interesante, actitud de los miembros, etc.).

\section{Resultados}

Hemos observado que esta actividad capacita a los alumnos para trabajar y liderar equipos de forma efectiva, de forma que se alcancen los objetivos comunes de un grupo de personas, contribuyendo al desarrollo personal y profesional de los mismos. Pone en acción a los estudiantes en situaciones comunicativas cercanas a la realidad profesional, que se basa en gran medida en el trabajo en equipo y la colaboración, así como promueve el pensamiento crítico y la creatividad. Los resultados de aprendizaje que se esperan después de la actividad son los siguientes:

1. Comprometerse en la realización de tareas colectivas

2. Aceptar, cumplir y proponer objetivos

3. Planificar, programar y distribuir tareas

4. Realizar las tareas que le son asignadas

5. Compartir conocimientos, compromiso y responsabilidad

6. Afrontar conflictos

7. Comunicarse eficazmente

\subsection{Competencia transversal CT8: Comunicación efectiva}

En relación a la competencia transversal CT8: Comunicación efectiva, los alumnos en general toman conciencia de la importancia de escribir correctamente y de los requisitos de una buena presentación. Se sienten motivados al versar su trabajo sobre un tema que les resulta de interés. Mediante la tarea de redacción del texto para la presentación, la exposición oral y la presentación del vídeo se comunican de manera formal, tanto oral como escrita. Por otro lado, la actividad de chat les permite comunicarse de manera relajada con un fin definido. A pesar de las bromas, y de los típicos errores al teclear, se observa que tienen interés en que su mensaje esté escrito correctamente. No recurren, sorprendentemente, al texting y cuando se dan cuenta de que algo lo han escrito mal, intervienen inmediatamente para rectificar. También piden disculpas cuando son conscientes de que lo que han formulado no es correcto pero no saben cómo mejorarlo. Se detectan errores de vocabulario, uso de tiempos verbales y formación de preguntas (que es un tipo de construcción al que deben recurrir frecuentemente para la planificación y reparto de tareas). Comprobar que los errores para formular preguntas son generalizados conduce al profesor a trabajar este aspecto en otras sesiones del curso.

2016, Universitat Politècnica de València

Congreso In-Red (2016) 


\subsection{Competencia transversal CT6: Trabajo en equipo y liderazgo}

\subsubsection{El trabajo en equipo}

Relacionado con la competencia transversal CT6: Trabajo en equipo y liderazgo, pasaremos a continuación a analizar los resultados de aprendizaje obtenidos a través de la actividad de chat directamente relacionados con el trabajo en colaborativo.

En cuanto a los dos primeros, comprometerse en la realización de tareas colectivas y aceptar, cumplir y proponer objetivos, podemos afirmar que los miembros de los grupos son conscientes de que tienen que cooperar en el trabajo y llevarlo a buen término. En general, el resultado 4, Realizar las tareas que le son asignadas, ha sido satisfactorio, pero ha habido excepciones. Por ejemplo, algunos alumnos no han realizado las tareas asignadas o no lo han hecho satisfactoriamente para el resto de los miembros de su grupo, o lo han hecho fuera de los plazos acordados por el grupo.

Donde hemos detectado más debilidades ha sido en la consecución del resultado 3, planificar, programar y distribuir tareas. Algunos grupos no han sido eficaces a la hora de programar, distribuir tareas y acordar aspectos formales de la presentación, muchas veces porque el líder no ha actuado como tal. Como observación general, la planificación ha consistido en acordar el tema y repartirse las tareas, trabajando ya cada alumno por separado durante el resto de la actividad. Ni siquiera la presentación oral ha implicado en algunos casos un auténtico trabajo en equipo y de colaboración. Para la planificación y reparto de las tareas, los grupos han utilizado diferentes estrategias. Una de ellas consiste en que todos los miembros del grupo participan en la toma de decisiones y no existe un líder:

(1) A: Who wants to search the advantages and the disadvantages of AMD?

B: And who from Intel?

C: I do

A: $\mathrm{ME}$

B:Bravo

A: okey I will search of intel

Se busca la opinión y el consenso de todos los miembros:

(2) A: Hi Team, about the topic I think we can do about SSD vs hard disc, we can do easier becouse is easy to find information about this issue

A: we know more thinks about this subject and the different are very different

A: I'm open mind about the others issue

B: Hello, I watched the different alternatives, and I think this issue is the best for us
A: because*
B: Let's wait Pablo, and read what he says

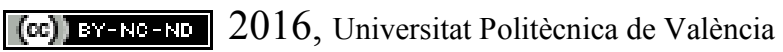


B: writes*

Cada miembro del grupo, una vez acordado el tema sobre el que versará la presentación del grupo, los contenidos a incluir y el reparto de tareas, actúa de manera independiente incorporando contenidos al documento PPoint, dejando para más adelante la labor de darle forma definitiva.

(3) A: i can search background infomation

B: ok

B: Then laura and me will focus on advantages and disadvantages of intel and AMD

(4) A: There's a lot of information around it so if you find anything just copy it on the drive document and we will polish it later.

En otra estrategia de grupo, resulta más destacada la figura del líder. En estos casos, es el líder quien organiza el reparto de tareas, recomienda los contenidos y fija el modo de trabajar:

(5) A: One of us search information about Intel, another about AMD and the last start organizing the presentation. We should start to search information about the topic. Maybe we should speak about the strategies the each company use to develop their own processor.

(6) A: What i usually do is save all the information and when the presentation is ready work in a document where we got all the information extended.

En cuanto a los resultados de aprendizaje 5 y 7, compartir conocimientos, compromiso y responsabilidad y comunicarse eficazmente, resulta interesante observar que los miembros del grupo se ayudan entre sí. Cuando alumnos con mejor nivel detectan que un compañero se ha expresado incorrectamente, le proporcionan la formulación correcta. En ocasiones, para que no haya malas interpretaciones, puntualizan que lo hacen no para alardear sino por si les ayuda. Esta actitud es reflejo de que el grupo comparte, se comunica y se ayuda para que los miembros mejoren:

(7) A: I found diferents advantages and disadvantages, I write it at the document, but only ideas

A: I think we need it

B: You mean you'll write it

A: yes, thanks Victor ;)

B: Not to be too nickpicky, just trying to correct.

C: yes

A: ok

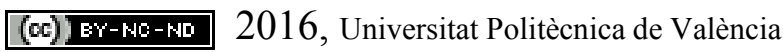

Congreso In-Red (2016) 
También lo hacen en relación con los contenidos de la presentación:

(8) A: I think we can improve the "Advantages of HDD" slide.

En cuanto al resultado 6, afrontar conflictos, no procede evaluarlo en esta actividad.

\subsubsection{El liderazgo}

La elección del líder se realiza una vez presentadas las instrucciones para realizar las tareas y después del primer contacto entre todos los miembros del grupo en el chat. Se observa una cierta reticencia a desempeñar el papel del líder del grupo que se suele asociar con una carga adicional de trabajo. Normalmente este papel recae en el miembro del grupo que menos reticente es; raras veces alguien se presenta como voluntario mostrando entusiasmo hacia la tarea. Por otro lado, el líder es casi siempre un alumno, a sugerencia de las alumnas. A continuación se pueden leer algunos intercambios en el chat:

9) A: who is the líder?

B: josé!!! You are the boy in this group

10) A: I don't want to be the leader. Antonio you are the leader

11) A: Any volunteer?

B: I don't think so. Wait until Juan is connected (...) who's going to be the leader?

12) A: Pedro, do you want to be the leader??

A: the leader must work more than the rest.
A: :D
B: mucho more!!

En general, el líder asume con facilidad su papel como el coordinador del grupo. Con frecuencia su actuación consiste en:

- preguntar por las opiniones de los demás,

- acotar el tema de la conversación,

- $\quad$ sugerir posibles temas,

- valorar las opiniones de los demás,

- ayudar a encontrar un consenso.

Se observa que el líder tiende a ser flexible y abierto a las opiniones de los demás. En la mayoría de los casos, la intervención del líder no resulta impositiva sino que siempre se produce a través de sugerencias o propuestas o se mitigan las imposiciones:

13) i think that we can do for example "differences between laptop and desktop computer".

(cc) EY-NG-ND 2016, Universitat Politècnica de València

Congreso IN-RED (2016) 
14) A: ok de los rios are going to be our designer and for example our principal presenter, are you agree?

A: and angel huerta you will defend artifical intelligence and attack human intelligence, ok?

En algunos casos, se ha podido detectar intentos de compensar la falta de liderazgo por parte de otro miembro del equipo con una expresa indicación de quién tiene que hacer el trabajo del líder:

15) A: i'm doing the task for the leader but i-m not the leader...

A: -.-

A: which is the topic?

B: okey then just my secretary

B: haha

B: (;)

B: ok. boss what we have to do?

El objetivo es pautar las etapas del trabajo, buscando la eficacia:

16) A: One of us search information about Intel, another about AMD and the last start organizing the presentation

A: Is that ok?

17) A: So, I believe the topic we should tackle is Windows vs linux.

B: ok

A:Do you agree or do you have any other preference?

Las conversaciones en el chat reflejan que se realiza un intenso trabajo colaborativo a la hora de elegir el tema de la presentación: se sugieren posibles temas, se intercambian opiniones, se presentan resultados de búsqueda en internet, se negocia el tema, se debaten sus ventajas y desventajas. Asimismo, se realiza una puesta en común en cuanto a la estructuración de la presentación y de quién tiene que encargarse de cada parte. Una vez dividido el trabajo, sin embargo, la colaboración entre los miembros del grupo disminuye considerablemente tal y como lo reflejan los chats. De hecho, las conversaciones se reducen, ya que los alumnos trabajan individualmente sobre su parte de la presentación. De los chats analizados, la mayoría ha bajado la intensidad de las conversaciones al 50\%-20\% en relación con las conversaciones anteriores cuando se negociaba el tema y el reparto de las tareas. Resulta lógico que la intensidad de las conversaciones disminuya, ya que muchos de los puntos pendientes de acordar ya están resueltos. Por otro lado, sin embargo, se observa la necesidad de colaboración continua entre los alumnos para que el resultado final, es decir la presentación, sea exitoso y que no resulte en algo fragmentado, desunido e incongruente.

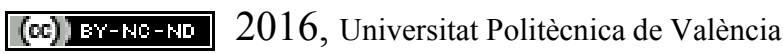

Congreso In-Red (2016) 


\section{Conclusiones}

En resumen, se constata una necesidad clara de reforzar el trabajo colaborativo entre los alumnos para que deje de ser de tipo cooperativo a partir del reparto de las tareas. Aunque la figura del líder tiene una interpretación diferente en el trabajo colaborativo, es decir todos los miembros de un grupo asumen la función del líder, creemos que en casos de colaboración en línea y desde lugares alejados geográficamente es necesaria una coordinación expresa por parte de una persona. Asimismo, opinamos que aquellas características negativas asociadas con la imagen de un líder (ej. ser autoritario, imponer una jerarquía, ser hombre, cargar con más trabajo, tomar decisiones en solitario, etc.) deberían cambiarse en la percepción de los alumnos. El concepto del liderazgo moderno se basa en ser flexible, abierto, participativo y ágil. Tener la mente abierta, cumplir la función del facilitador, saber tomar riesgos, ser adaptable y sociable, así como dejar de lado la jerarquía son destrezas que los alumnos hoy en día deben si no aprender y desarrollar, al menos ser conscientes de ellas. Sólo entendiendo la función de un líder de esta manera, podemos salvar las dificultades que pueden presentarse en el trabajo colaborativo de nuestros alumnos.

A pesar de la importancia del líder para un trabajo en equipo eficaz, observamos que no se menciona explícitamente al líder en los recursos de aprendizaje de CT8. Sin embargo, puede afirmarse que la práctica de las habilidades relacionadas con un buen líder sin duda capacitará a nuestros alumnos para el desempeño de sus tareas profesionales futuras. Con el fin de concienciar a los alumnos sobre el papel clave de un líder en el trabajo colaborativo proponemos realizar actividades que refuercen el concepto del liderazgo moderno, por ejemplo a través de 'storytelling' donde los alumnos narran la historia de un líder conocido y exitoso, debatiendo y comparando las cualidades de un líder antiguo y moderno, o realizando un role-play para que los alumnos puedan poner en práctica las destrezas de un líder moderno. Pensamos que este tipo de entrenamiento puede contribuir a que perciban el rol del líder como necesario y desprovisto de connotaciones negativas.

\section{Referencias}

BuRns, A. (1999). Collaborative Action Research for English Language Teachers. Cambridge: Cambridge University Press.

CARRIÓ PASTOR, M.L. (ed.) (2006). Aprendizaje colaborativo asistido por ordenador. Valencia: Blau Verd.

CARRIÓ PASTOR, M.L. (2008). "Learner-instructor collaborative design of content and language integrated writing activities" en ITL - International Journal of Applied Linguistics, vol. 156, p. 265-276.

CARRIÓ PASTOR, M.L. (2009). "Enhancing learner-teacher collaboration through the use of on-line activities", en I. González-Pueyo, C. Foz-Gil, M. Jaime Siso \& M. J.

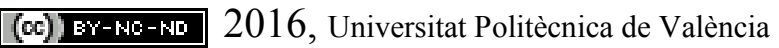


Luzón Marco, (eds.), Teaching Academic and Professional English Online. Berlin: Peter Lang.

CARrió-PASTOR, M.L. y Perry, D. (2010). "The Collaborative Approach in Content and Language Integrated Learning”, en Revista Alicantina de Estudios Ingleses, vol. 23, p. 69-81.

CARrió-PASTOR, M.L. y SKORCZYNSKA, H. (2015). "Collaborative learning and communication technologies in teaching business English" en Procedia - Social and Behavioral Sciences, vol. 178, p. 32-37.

Chung, J. (1991). "Collaborative learning strategies: The design of instructional environments for the emerging new school” en Educational Technology vol. 32(12), p. 15-22.

JONES, A. y IsSROFF, K. (2005). "Learning technologies: Affective and social issues in computer-supported collaborative learning" en Computers \& Education vol. 44, p. 395-408.

Kessler, G., BiKowsKi, D. y BogGs, J. (2012). “Collaborative writing among second language learners in academic web-based projects" en Language Learning \& Technology vol. 16(1), p. 91-109.

LiAW, S.-S., CHEN, G-D. y HuANG, H.-M. (2008). "User's attitudes toward Web-based collaborative learning systems for knowledge Management" en Computers \& Education vol. 50, p. 950-961.

MCCAfferty, S.G., JacobS, G.M. y DaSilva IddingS, A.C. (2006). Cooperative Learning and Second Language Teaching. Cambridge: Cambridge University Press.

NunAN, D. (ed.) (1992). Collaborative Language Learning and Teaching. Cambridge: Cambridge University Press.

ROMNEY, J.C. (1997). "Collaborative learning in a translation course" en Canadian Modern language Review vol. 54, p. 48-67.

Roschelle, J. y TeAsley S. D. (1995). "Construction of shared knowledge in collaborative problem-solving" en C. O'Malley, (ed.), Computer Supported Collaborative Learning. Berlin: Springer-Verlag: p. 69-97.

STORCH, N. (2005). "Collaborative writing: Product, process, and students' reflections" en Journal of Second Language Writing vol. 14, p. 153-173.

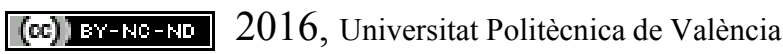

Congreso In-Red (2016) 